\title{
Treatment of Primary Liver Tumors and Liver Metastases, Part 2: Non-Nuclear Medicine Techniques
}

\author{
Francois H. Cornelis ${ }^{1,2}$ and Stephen B. Solomon ${ }^{1}$ \\ ${ }^{1}$ Department of Radiology, Memorial Sloan Kettering Cancer Center, New York, New York; and ${ }^{2}$ Sorbonne Université, Department of \\ Radiology, Tenon Hospital, Paris, France
}

Learning Objectives: On successful completion of this activity, participants should be able to (1) describe the mechanisms of actions of the most used ablation techniques; (2) discuss the potential advantages and disadvantages of each thermal ablation modality; (3) discuss the differences between current transarterial therapies; and (4) describe the rationale and treatment selection of the appropriate locoregional modality for the specific clinical indications.

Financial Disclosure: The authors were supported in part through the NIH/NCI Cancer Center Support Grant P30 CA008748. Dr. Solomon received research grants from AngioDynamics and GE Healthcare; serves as a consultant for Medtronic, Inc., Johnson and Johnson, BTG, XACT, and Adgero; and has investment interest in Johnson and Johnson. The authors of this article have indicated no other relevant relationships that could be perceived as a real or apparent conflict of interest.

CME Credit: SNMMI is accredited by the Accreditation Council for Continuing Medical Education (ACCME) to sponsor continuing education for physicians. SNMMI designates each JNM continuing education article for a maximum of 2.0 AMA PRA Category 1 Credits. Physicians should claim only credit commensurate with the extent of their participation in the activity. For CE credit, SAM, and other credit types, participants can access this activity through the SNMMI website (http://www.snmmilearningcenter.org) through December 2021.

Image-guided procedures are used worldwide in the management of primary liver tumors and liver metastases. These locoregional therapies include local tumor ablation and transarterial therapies and can occasionally downstage an inoperable patient to an operable status. In hepatocellular carcinoma, data have suggested that for tumors smaller than $2 \mathrm{~cm}$ ablation may be preferable to surgery. Similar results are emerging for colorectal cancer liver metastases. Catheter-directed therapies such as bland embolization, transarterial chemoembolization, and drug-eluting beads represent potential techniques that can provide survival benefit for inoperable patients. In this review we highlight the most used techniques and the evidence supporting their current indications for the treatment of liver tumors. We briefly review upcoming developments in combination strategies with temperature-sensitive liposomes or immunotherapy for enhancing ablation efficacy.

Key Words: liver; hepatocellular carcinoma; metastasis; ablation; embolization

J Nucl Med 2018; 59:1801-1808

DOI: 10.2967/jnumed.116.186379

$\mathbf{I}$

nterventional oncology (IO) refers to image-guided procedures that allow the treatment of cancer under minimally invasive conditions (1). Since the early 1980s, IO approaches have been proposed for primary and secondary liver tumors and have become increasingly common in clinical practice. Liver ablation procedures and catheter-directed liver therapies are currently used worldwide to manage liver tumors (2). Classification systems such

Received Feb. 2, 2018; revision accepted Oct. 19, 2018.

For correspondence or reprints contact: Stephen B. Solomon, Department of Radiology, Memorial Sloan Kettering Cancer Center, 1275 York Ave., New York, NY 10065.

E-mail: solomons@mskcc.org

Published online Oct. 25, 2018.

COPYRIGHT (c) 2018 by the Society of Nuclear Medicine and Molecular Imaging. as the Barcelona Clinic Liver Classification help to determine the use of IO procedures and to distinguish the selection of one IO procedure over another $(3,4)$. However, selection is not always made according to guidelines but is often dependent on the availability and experience of interventional oncologists as well as access to the different technologies (5). The lack of standardization in treatment selection renders comparison of research studies difficult and hampers reproducibility. This review offers a critical discussion of IO procedures for liver tumors and their outcomes and includes future perspectives.

\section{PERCUTANEOUS IMAGE-GUIDED ABLATION FOR LIVER TUMORS}

\section{Rationale and Treatment Selection}

Percutaneous ablation techniques can be performed with curative intent for primary and secondary liver tumors. Several ablation methods are known to have tumoricidal effects: chemical injection of alcohol, heating, cooling, and irreversible electroporation or electrochemotherapy (5-8). Under imaging guidance such as ultrasound, CT, MRI, or PET/CT, percutaneous ablation can be performed effectively (Fig. 1) (1). The following describes the ablation procedure. First, an applicator specific to each ablation technique is inserted percutaneously into the targeted tissue under imaging guidance. Applicators vary in diameter (13-17 gauge) and shape (straight needle to umbrella) (Fig. 2). The ablation modality, needle shape of the applicator, and amount of energy locally delivered affect the volume of ablation. Often, a maximum long axis of $4-5 \mathrm{~cm}$ and a large ablation zone of $2-3 \mathrm{~cm}$ are observed for a single applicator. Several other factors also affect the volume or the effectiveness of the ablation. Properties of the local tumor environment, including water content and presence of cirrhosis, influence thermal conductivity, and proximity to large vessels leads to a heat sink effect (9).

Thermal ablation procedures are the most frequently used procedures for liver tumors. These include radiofrequency ablation (RFA) and microwave ablation (MWA) and, to a lesser extent, cryoablation. Their specific advantages and limitations are shown in Table 1 (7). 


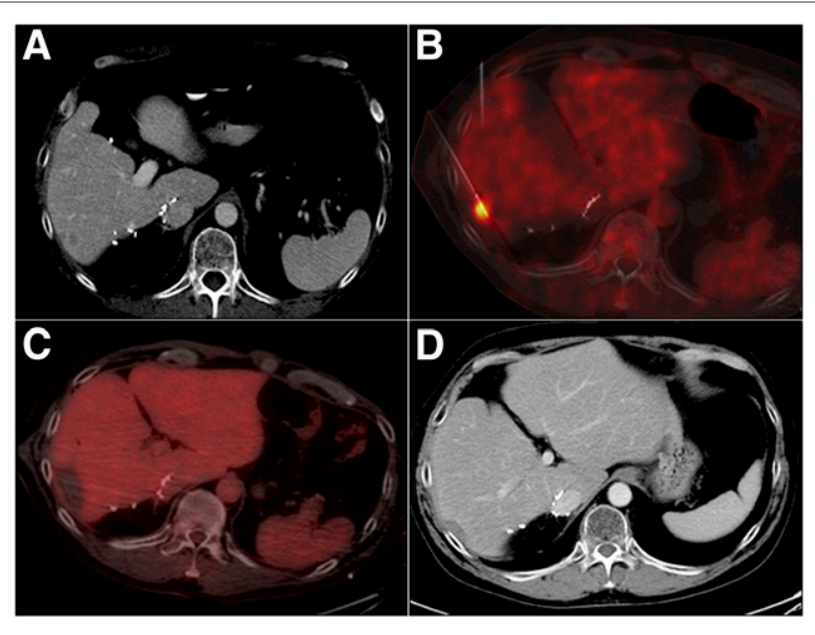

FIGURE 1. Percutaneous PET/CT-guided RFA of liver metastasis of colorectal cancer. (A) 54-y-old man with metastatic colorectal cancer presenting with arterially enhancing $1.3 \times 1.0 \mathrm{~cm}$ lesion with washout in segment 5. (B and C) PET/CT-guided MWA using split-dose technique for targeting tumor $(B)$ and for immediate assessment of ablation completeness (C). (D) Follow-up contrast-enhanced CT imaging was performed 8 wk after ablation and every 2-4 mo thereafter, showing continuous involution of ablation zone $2 \mathrm{y}$ after ablation.

All achieve substantially different ablation zones and ablation margins (10). We previously reviewed these procedures in comparison with irreversible electroporation, a relatively new, nonthermal ablation technique (7). During RFA and MWA, the interventional oncologist delivers electric or electromagnetic energy, respectively, causing the temperature to rise within the targeted tissue. Although resistive heating is generated by a high-frequency oscillating electric current during RFA, an oscillating electric field causes polar molecules (primarily water) to continuously realign and rise in kinetic energy during MWA (5). Both procedures can cause up to a 3-fold elevation in tissue temperature (from $37^{\circ} \mathrm{C}$ to $\geq 100^{\circ} \mathrm{C}$ ). Targeted cancer cells that are exposed to sustained elevated temperatures are directly or indirectly killed via coagulative necrosis wherein the injured tissue undergoes protein denaturation and cell membrane dysfunction. The heating also causes desiccation of the tissue and destruction of microvasculature. Conversely, cryoablation causes the temperature to dip below $-40^{\circ} \mathrm{C}$ within the targeted tissue. Cryoablation uses expanding argon gas to induce a freeze-thaw cycle in targeted lesions, resulting in crystal formation and osmotic shock and ultimately cell death in a small radius around the probe. Recently, MWA has been gaining more interest and acceptance for liver ablation as it has the potential to improve energy control and allow increased delivery of energy compared with RFA (6). MWA has less dependency on the electric conductivities of tissue, and energy delivery is less limited by the exponential rising electric impedances of tumor tissue (11); therefore, it produces higher intratumoral temperatures, larger ablation volumes, and faster ablation times. MWA also leads to a decreased heat sink effect.

To date, the ideal candidate for tumor ablation is a patient with paucifocal disease, a single tumor up to $5 \mathrm{~cm}$ or up to 3 tumors smaller than $3 \mathrm{~cm}$ (very early or early stages according to the Barcelona Clinic Liver Classification staging system), and tumors $1 \mathrm{~cm}$ away from major bile ducts and high-flow vessels (2). An ablation margin of at least $5 \mathrm{~mm}$ is recommended because it has been associated with the best local tumor control, although most would advocate for a larger margin for metastases $(>10 \mathrm{~mm})(12)$. Although a single applicator is more often used to achieve this objective, well-positioned overlapping ablations using multiple, simultaneously activated electrodes or probes can be used to increase the ablation volume and ensure the efficiency of ablation. Tumors larger than $3 \mathrm{~cm}$ and those with complex geometry or near surrounding organs or sensitive tissue are more difficult to treat and call for alternative strategies (13). A recent singlecenter study assessed the use of a high-power, multiantenna MWA device to treat tumors larger than $3 \mathrm{~cm}$. This MWA device showed primary technique effectiveness rates of $91.6 \%$ overall, $93.7 \%$ in tumors $4 \mathrm{~cm}$ or smaller, and $75 \%$ in tumors larger than $4 \mathrm{~cm}(14)$.

\section{Clinical Outcomes for Primary Liver Tumors}

RFA was first introduced $20 \mathrm{y}$ ago, and its clinical outcomes are the most extensively documented compared with the other procedures in terms of local tumor response, progression rate, median survival, and 3-y survival (Table 2) (15).

Survival rates of RFA have been shown to be comparable to resection for primary liver tumors. Chen et al.'s study (16) analyzing 180 patients with solitary hepatocellular carcinoma (HCC) smaller than $5 \mathrm{~cm}$ found comparable overall survival rates at 1, 2, 3 , and $4 \mathrm{y}: 96 \%, 82 \%, 71 \%$, and $68 \%$, respectively, for ablation versus $93 \%, 82 \%, 73 \%$, and $64 \%$ for resection. A metaanalysis showed no difference between resection and ablation with respect to overall survival up to $4 \mathrm{y}$ for HCC $3 \mathrm{~cm}$ or smaller (17). On the

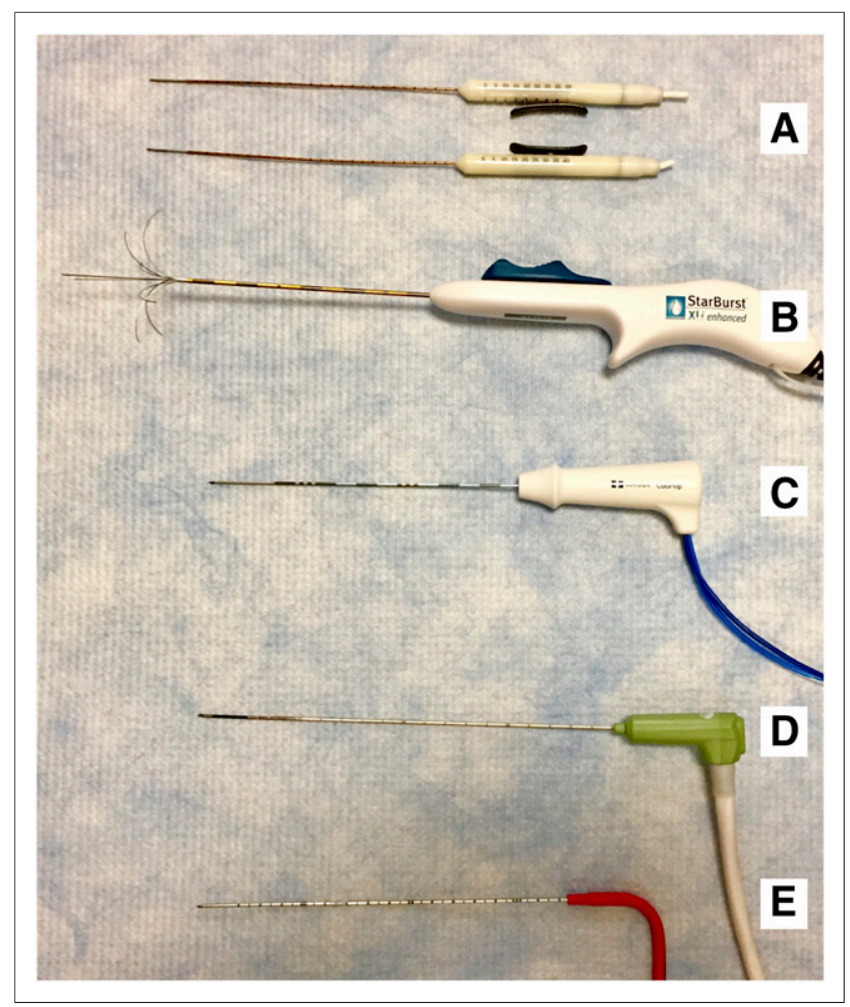

FIGURE 2. Ablation devices commonly used. (A) Nanoknife IRE probes, Angiodynamics. (B) Starburst RFA probe, Angiodynamics. (C) Valleylab CoolTip RFA probe, Covidien. (D) Certus 140 MWA, NeuWave Medical. (E) IceRod 140 cryoablation probe, Galil Medical. 
TABLE 1

Advantages and Limitations of Ablative Techniques Most Frequently Used in Liver

\begin{tabular}{|c|c|c|c|}
\hline Parameter & RFA & MWA & Cryoablation \\
\hline Mechanisms of action & Oscillating electric current (ions) & Dielectric hysteresis (water) & Joule Thompson (Argon gas) \\
\hline Range of temperature obtained & $\pm 100^{\circ} \mathrm{C}$ & $>100^{\circ} \mathrm{C}$ & $-170^{\circ} \mathrm{C}$ to $0^{\circ} \mathrm{C}$ \\
\hline Performance of imaging monitoring & Low & Mid (gas formation) & High (ice ball) \\
\hline Margins & Large & Sharp & Sharp \\
\hline Collagen & Injured & Injured & Respected \\
\hline Size of ablation & $<3 \mathrm{~cm}$ & $<3-5 \mathrm{~cm}$ & $<5 \mathrm{~cm}$ \\
\hline Mean length of procedure & $15 \min$ & $10 \mathrm{~min}$ & $30 \mathrm{~min}$ \\
\hline Cost & Low & Mid & High \\
\hline
\end{tabular}

other hand, in a later trial in 230 patients with $\mathrm{HCC} 5 \mathrm{~cm}$ or smaller or up to 3 nodules, each smaller than $3 \mathrm{~cm}$ (18), Huang et al. (18) found that resection may provide better overall survival rates; 1-, 2-, 3-, 4-, and 5-y survival rates for ablation were $87 \%$, $76 \%, 70 \%, 66 \%$, and $55 \%$, respectively, versus $98 \%, 96 \%, 92 \%$, $83 \%$, and $76 \%$ for resection. A study comparing RFA and MWA in 154 patients found that, for a median follow-up of 31 mo for RFA and 24 mo for MWA, the rate of local tumor progression was $17.7 \%$ with RFA and $8.8 \%$ with MWA (19). However, in a recent study evaluating the local efficacy and long-term outcomes of MWA in 142 HCC patients with 294 tumors, local tumor progression was observed in $44(15 \%)(20)$.

\section{Clinical Outcomes for Secondary Liver Tumors}

Comparisons between RFA and MWA for the treatment of liver metastases, most often colorectal liver metastases, have shown that both produce different clinical outcomes in local tumor progression (2\%-60\% for RFA vs. $9.6 \%-14.5 \%$ for MWA), median survival (10.9-60 mo for RFA and 9-41.8 mo for MWA), and 3-y survival rates $(37 \%-77 \%$ for RFA and $32 \%-70 \%$ for MWA) $(6,21,22)$.

\section{Complications}

The mortality rate for thermal percutaneous ablation is low, up to $0.1 \%$ for treatment of HCC (23). However, complications can occur (24). Major complications occur in about $2 \%-3 \%$ of procedures and include peritoneal hemorrhage, tumor cell seeding, intrahepatic abscess formation, bile duct injury, and bowel perforation (25). The most common minor complications are pain, skin burns, fever, biloma, hematoma, and pneumothorax. These complications justify further improvements using image guidance or protective techniques such as hydro- or carbon dioxide separation techniques to displace organs surrounding the ablation zone $(1,25)$.

\section{TRANSARTERIAL THERAPIES}

\section{Rationale and Treatment Selection}

Transarterial therapies consist of hepatic transarterial embolization (TAE) or transarterial chemoembolization (TACE) therapies. These therapies are often proposed with palliative intent when curative treatment is not possible. Transarterial therapies are the primary treatment for Barcelona Clinic Liver Classification stage B HCC tumor (asymptomatic, noninvasive, and multinodular). They are an optional treatment for cholangiocarcinoma and metastases of colorectal, neuroendocrine, breast, or melanoma cancer (2). In general, they are indicated for patients with unresectable disease involving less than $50 \%$ of the liver and well-compensated cirrhosis (Child-Pugh A or B) (26). Contraindications are advanced cirrhosis (Child-Pugh C) and poor functional status. Relative contraindications

TABLE 2

Outcomes After Percutaneous Liver Ablation

\begin{tabular}{lrr}
\hline \multicolumn{1}{c}{ Outcome } & RFA (long-term) & MWA (short-term) \\
\hline Local tumor progression & & $12.7(5-19.6)$ \\
\hline HCC & $14.4(6-26)$ & $11(9.6-14.5)$ \\
Metastasis & $20(2-60)$ & $93.1(85.7-98.2)$ \\
1-y overall survival & & $80.8(71.7-100)$ \\
HCC & $94(92-98)$ & $(83.9-84.9)$ \\
Metastasis & $65.6(37.7-82.3)$ & $78.3(49-77.9)$ \\
3-y overall survival & $50(37-77)$ & $50(32-70)$ \\
HCC & $2.7(0-5.7)$ & $3.9(2.7-4.1)$ \\
Metastasis & &
\end{tabular}

Data are mean percentage, followed by range in parentheses, for published outcomes $(6,15,26,16-19,22-25)$. 
are tumor within the main portal vein, biliary obstruction, and hepatic encephalopathy.

Transarterial therapies may yield a survival benefit in cases where they downstage the disease, placing the patient back within Milan/University of California San Francisco criteria eligibility for curative therapy such as liver transplantation or ablation (1 lesion $\leq 6.5 \mathrm{~cm}$ or $2-3$ lesions $\leq 4.5 \mathrm{~cm}$ with cumulative tumor burden $\leq 8 \mathrm{~cm}$ ) (27). They provide also a survival benefit of their own when compared with conservative measures (28). The rationale for these therapies is that the liver tumor preferentially receives blood $(\leq 95 \%)$ from the hepatic artery whereas normal tissue receives blood from the portal venous system (26). This allows for embolization and locoregional chemotherapy of the tumor without significantly damaging the surrounding healthy parenchyma.

Transarterial therapies involve the selective catheterization of a femoral or radial artery; the catheter is placed in the appropriate hepatic arterial branches that provide a blood supply to the tumor. Thereafter, selective embolization is performed whereby embolic agents are delivered via the catheter to induce tumor ischemic necrosis while limiting normal tissue ischemia (27). TAE uses various embolic agents, which may be spheric or nonspheric, resorbable or nonresorbable, calibrated or noncalibrated (e.g., polyvinyl alcohol and calibrated microspheres) (Figs. 3 and 4) $(29,30)$.

TACE combines the delivery of embolization agents with chemotherapy drugs - for example, doxorubicin, cisplatin, epirubicin, mitoxantrone, mitomycin $\mathrm{C}$, and irinotecan. Depending on the chemoembolization combination, a total of $10-100 \mathrm{mg}$ of drugs is administrated through the catheter into the tumor. The goal of TACE is to achieve a greater concentration of chemotherapy drugs in the vicinity of the tumor than is possible with intravenous administration. To do this, chemotherapy drugs are delivered into the blood vessels just before (conventional TACE) or at the same time as (drug-eluting beads [DEB]-TACE) embolization agents. Embolization-induced hypoxia promotes the secretion of vascular endothelial growth factor, which increases vessel permeability, ultimately causing cell membrane dysfunction and stasis of blood flow. This allows the drugs to accumulate in the tumor tissue while the decreased systemic circulation retains them at the local level (27).

Although TACE is not an uncommon procedure, considerable heterogeneity exists between centers and interventional radiologists. Conventional TACE includes the extemporaneous preparation of drugs as either a solution (for lipophilic drugs) or as a drug-Lipiodol (Guerbet) emulsion (26). Lipiodol, also known as ethiodized oil, can be very effective as a drug-carrying, tumorseeking, and embolizing agent. When injected, Lipiodol is incorporated by cancer cells by pinocytosis. On TACE-induced hypoxia within cancer cells, Lipiodol is retained within the cells, where it remains for months, whereas it is washed out from nontumor portions of the liver (31). Depending on the tumor size, vascularity, and fluoroscopic findings, a total volume of about $10-15 \mathrm{~mL}$ of Lipiodol is injected mixed with a similar volume of drug before a mixture of the embolic agent and a contrast medium is delivered.

DEB-TACE involves the injection of spheric embolic beads in the 40 - to $900-\mu \mathrm{m}$ range loaded with the chemotherapeutic drug, usually doxorubicin, into the tumor bed (32). DEBs are composed of a hydrophilic and ionic polymer. Drugs can be bound via an ion-exchange mechanism. This allows the drug to be delivered simultaneously on the embolic beads.

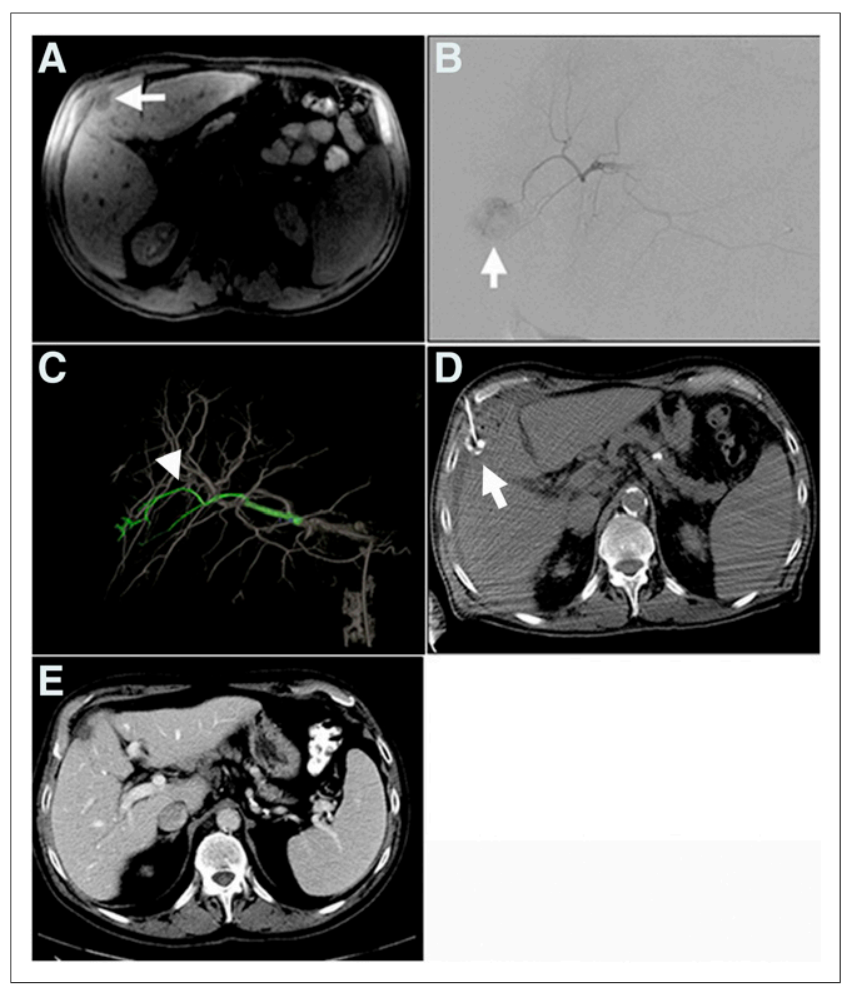

FIGURE 3. Transarterial embolization and percutaneous ethanol injection of HCC in 74-y-old man with hepatocellular in background of hepatitis $\mathrm{C}$ and cirrhosis. (A) MRI demonstrates solitary $2.2 \times 1.4 \mathrm{~cm}$ segment $4 \mathrm{~B}$ tumor. (B-D) Primary treatment of this tumor was performed by combined transcatheter embolization ( $\mathrm{B}$ and $\mathrm{C}$ ) and percutaneous ethanol injection (D). Segment 4 branch of left hepatic artery was identified as tumor feeding vessel by both DSA (B) and Flight Plan for Liver software $(C)$ and embolized to stasis using $1 \mathrm{~mL}$ of $40-120 \mu \mathrm{m}$ EMBOspheres. CT was performed at conclusion of procedure and used to target lesion for ethanol injection (D). (E) CT imaging follow-up demonstrates that good local tumor control achieved by combined technique is maintained after $14 \mathrm{mo}$.

\section{Clinical Outcomes for Primary Liver Tumors}

To date, the literature shows that both TAE and TACE confer survival benefit compared with conservative management in patients with unresectable HCC. A large retrospective study in 322 patients showed that patients who underwent TAE experienced a median survival of 21 mo (33). When TAE with gelatin sponge particles, conventional TACE (using doxorubicin), and conservative treatment were compared in 112 HCC patients, 1- and 2-y survival probabilities were $75 \%$ and $50 \%$ for TAE, $82 \%$ and $63 \%$ for TACE, and $63 \%$ and $27 \%$ for conservative management, respectively (TACE vs. conservative management, $P=0.009$ ) (34). Similarly, a metaanalysis in 503 patients demonstrated a significant 2-y survival benefit for TACE compared with conservative management (odds ratio, 0.53 [range, 0.32-0.89], $P=0.017$ ) (28).

Several studies have compared conventional TACE with DEBTACE. No differences in complete response, overall response, or disease control rates were observed, but side effects were lower with DEB-TACE (35). Comparison of DEB-TACE and TAE in HCC patients found no difference in time to progression, progression-free survival, or overall survival (30). The median survival time remained similar, both at around 20 mo. 


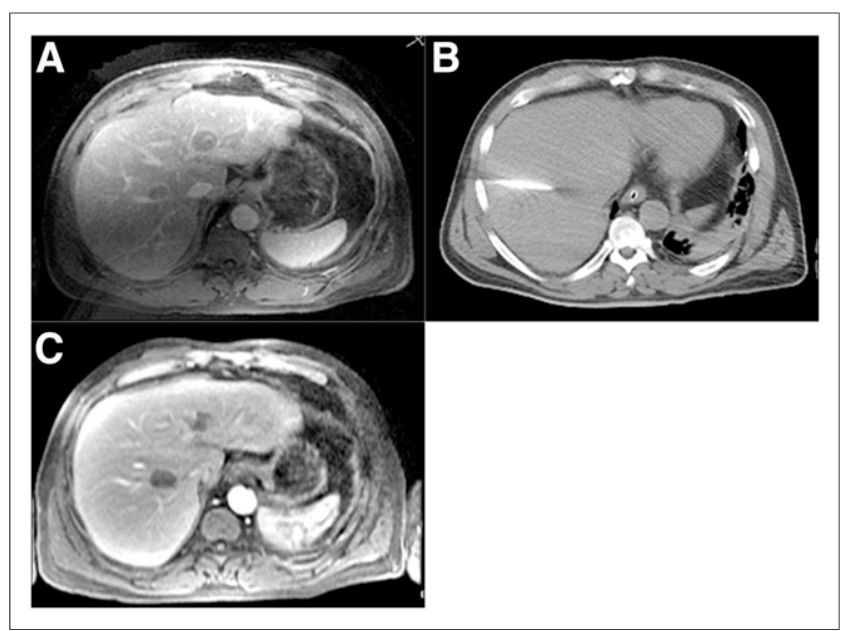

FIGURE 4. Transarterial embolization and percutaneous MWA of HCC in 63-y-old man with HCC. (A) MRI of liver identifies mildly hyperenhancing $2.5 \times 2.1 \mathrm{~cm}$ lesion in segment $4 \mathrm{~A}$ displaying mild T2 hyperintensity, with washout and $2.2 \times 1.4 \mathrm{~cm}$ lesion in segment 8 anterior to right hepatic vein also displaying washout, both suggestive of HCC. Segment $2 / 3$ and segment 4 hepatic arteries, identified as branches feeding segment 4 tumor, were embolized to stasis using $2 \mathrm{~mL}$ of 40-120 $\mu \mathrm{m}$ EMBOspheres (also ethanol). (B) Segment 8 tumor was not identified angiographically but was visible on ultrasound and was treated with MWA. (C) MRI follow-up at 3 mo shows stable postembolization (and postalcohol ablation) scar in hepatic segment $4,1.9 \times 1.3 \mathrm{~cm}$, and stable post-MWA scar in hepatic segment $7 / 8,2.6 \times 1.7 \mathrm{~cm}$.

TACE has been shown to be effective for unresectable intrahepatic cholangiocarcinoma, with disease control rates of $85 \%-$ $95 \%$ at $3 \mathrm{mo}$, time to progression of 6-14 mo, and median overall survival of $12-13$ mo (36-38).

\section{Clinical Outcomes for Secondary Liver Tumors}

TACE may also treat colorectal liver metastasis, neuroendocrine, and breast tumors. Various patient selection approaches and techniques have been published for the treatment of colorectal liver metastasis $(39,40)$. Per lobe, 2 treatment sessions spaced at 3-4 wk with delivery of $100 \mathrm{mg}$ of irinotecan at each session are recommended. For patients with bilobar disease, each lobe is therefore treated twice. Lobes are treated alternatively every $2 \mathrm{wk}$. For tumor involvement of less than $60 \%$, TACE seems to be achieving a better outcome than supportive care, especially in cases of hypervascular metastases (41). In a multiinstitutional study in patients with colorectal liver metastasis, overall survival after DEB-TACE with irinotecan was 22 mo and progression-free survival was 7 mo (42). For neuroendocrine tumors, conventional TACE and DEB-TACE thus far have similar results whatever drugs are used. The median time to progression was $15-18$ mo and the 2-y survival was $66 \%-100 \%$ (43-45). Lastly, for breast cancer liver metastasis, the median overall survival of patients who received TACE with doxorubicin was 7.3$47.0 \mathrm{mo}$ (46), the median disease-free survival was $2.9-17 \mathrm{mo}$, and response rates were $7 \%-73.5 \%$. Overall, for liver metastases, TACE may be a valuable tool in the management of surgical candidates by downsizing the tumors in a neoadjuvant setting and for early conversion to resectability without adverse effects (47).

\section{Complications}

Transarterial therapies are generally considered to be well tolerated, with major complications occurring in only $4 \%-7 \%$ of procedures and a 30-d mortality rate of approximately $1 \%$ (48). Targeted injection of DEB-TACE allows higher cytotoxic drug concentrations within the tumor and lower systemic exposure due to controlled drug-release over several days within the treated tissue (31), lessening the risk of adverse events compared with conventional TACE (35). The most common serious complications of TACE are liver abscess or liver infarction and cholecystitis ( $\sim 2 \%$ of patients).

A very common complication of transarterial therapies is postembolization syndrome, which occurs in up to $90 \%$ of TACE patients and lasts for up to $3 \mathrm{~d}$ (48). Symptoms consist of fever, nausea, vomiting, and abdominal pain (49). This is thought to result from therapeutic cytotoxicity, tumor ischemia, and resulting intrahepatic and extrahepatic inflammation occurring after embolization (50). Combination therapy is useful to manage the symptoms $(49,51)$. Prolonged pain may suggest that embolization has occurred in nontargeted organs, in particular the pancreas, resulting in pancreatitis, or the gallbladder or upper gastrointestinal tract, resulting in cholecystitis or gastric or duodenal ulceration. This is a serious complication. The gallbladder usually tolerates unintended embolization; however, to avoid this complication, treatment has to be performed distal to the cystic artery (2). Further research is needed to understand the impact of the primary disease site, TACE technique, and chemotherapeutic agent on postembolization symptoms.

Other serious complications may occur, including liver failure, tumor rupture ( $<1 \%$ of cases) or biloma, and vessel injury. TACE is infrequently associated with bone marrow suppression and alopecia. With varying frequency, each intraarterial therapy is associated with the risk of dissection and occlusion, which can render further procedures more challenging.

\section{Other Implications for Patient Care}

Percutaneous liver ablations are generally performed under conscious sedation. In complex cases, as well as in cases where breathhold is required for imaging (e.g., PET-guided liver ablation), general anesthesia and monitoring is required. Transarterial therapies can be performed under conscious sedation or general anesthesia. An antiemetic (palonosetron hydrochloride, $0.25 \mathrm{mg}$ intravenously) and antibiotic (cephazolin, $1 \mathrm{~g}$ intravenously) are administered before the procedure. The patients recover in the postanesthesia recovery unit, are observed overnight and discharged from the hospital the following day (percutaneous ablation) or after an average of $3 \mathrm{~d}$ (hepatic tumor embolization).

\section{Recommended Clinical and Imaging Follow-Up}

Patients are evaluated with multiphase CT and PET (for avid tumors) 4-8 wk after a successful IO procedure and every 2-4 mo thereafter for the following $2 \mathrm{y}$. MRI will be used instead of dynamic liver CT for patients with contraindication to contrast agents or those with increased creatinine levels. MRI is also acceptable for certain sarcoma or melanoma tumors.

Clinic visits are scheduled at 1-2 wk after embolization or ablation-with laboratory tests including liver function tests, complete blood counts, prothrombin time, and international normalized ratio-and at the time of imaging follow-up thereafter. For patients without local tumor progression for $2 \mathrm{y}$ after treatment, subsequent follow-up can be done at 6-mo intervals, in perpetuity.

\section{COMBINED THERAPIES AND FUTURE PERSPECTIVES}

To optimize outcomes for patients with 3- to 5-cm tumors, percutaneous ablation may be performed in combination with TAE/TACE. 
This takes advantage of 2 different tumoricidal methods to ensure adequate tumor destruction. Moreover, arterial embolization and subsequent tumor contrast uptake may improve tumor visualization and increase the efficiency of ablation by reducing heat sink and thus increasing the rate of heating and ablation size. When ablation follows embolization, it is possible to target areas of tumor showing lower postembolization contrast uptake resulting from less deposition of embolic material and in which the likelihood of viable residual tissue is higher (Fig. 3). Furthermore, the drug delivered during TACE may increase the sensitivity of tumor cells to elevated temperatures, potentially enhancing the efficacy of the ablation (Fig. 4) (13).

In a study including 139 patients with recurrent $\mathrm{HCC}$ after resection, the 1-, 3-, and 5-y overall survival rates were 94\%, 69\%, and $46 \%$ for the combination-therapy group (RFA+TACE) and $82 \%, 47 \%$, and $36 \%$ for the RFA-alone group (52). Improved overall survival was observed in patients with 3- to 5-cm tumors. In another study in $189 \mathrm{HCC}$ smaller than $7 \mathrm{~cm}$, overall survival and PFS rates were significantly better in the embolization-ablation group than in the group who underwent RFA alone (53). The overall survivals were $92.6 \%, 66.6 \%$, and $61.8 \%$ for the TACE-RFA group and $85.3 \%, 59 \%$, and $45.0 \%$ for the RFA group, at 1-, 3-, and 4-y, respectively $(P=0.002)$. The recurrence-free survivals were $79.4 \%$, $60.6 \%$, and $54.8 \%$ and $66.7 \%, 44.2 \%$, and $38.9 \%$, respectively $(p=$ 0.009). Recent studies have demonstrated that patients with large, unresectable HCC experienced significantly longer survival with combination therapy using both RFA/MWA and TAE/TACE when compared with patients who had undergone either TACE or MWA alone; however, there is still a lack of studies comparing different combined embolization and ablation strategies (6).

Targeted drug delivery through activatable nanocarriers such as liposomes is a promising further development (54). The physiochemical properties of liposomes can be adapted for IO by altering their composition, size, shape, and surface properties. The overall goal of using these nanocarriers in drug delivery is to treat a disease more effectively by providing an increased amount of drugs with minimum side effects. They also might enhance the efficacy of thermal ablation. Several other nanocarriers have already been developed; however, only a few of them are clinically approved for the delivery of antitumor drugs for their intended actions into liver and none are approved for use by IO techniques. Recently, in a phase III clinical trial, lyso-thermosensitive liposomal doxorubicin was safely used to locally release a high concentration of doxorubicin after RFA for HCC lesions larger than $5 \mathrm{~cm}$ to improve efficacy (55).

Although the goals of tumor ablation by IO are similar to resection, ablation differs in that the tumor material is left in situ. Thus, similar to radiation therapy, ablation may trigger a systemic antitumor immune response whereas surgery would not $(56,57)$. Accumulating evidence has shown that thermal ablation can induce distant tumor regression or progression (58). This abscopal effect may depend on the activation of the antitumor immune response (Fig. 5). It may be spontaneous or directed by immunotherapies and checkpoint inhibitors (59). After thermal ablation, various immune modulating factors such as tumor antigens, danger signals, and cytokines are released or expressed $(60,61)$. However, as this immune response is thought to be weak, combination strategies are being developed to increase antigen presentation to T-cells or enhance the T-cell function for cancer cell elimination by blocking the inhibition of cytotoxic T-lymphocytes (62). Although most of these approaches currently

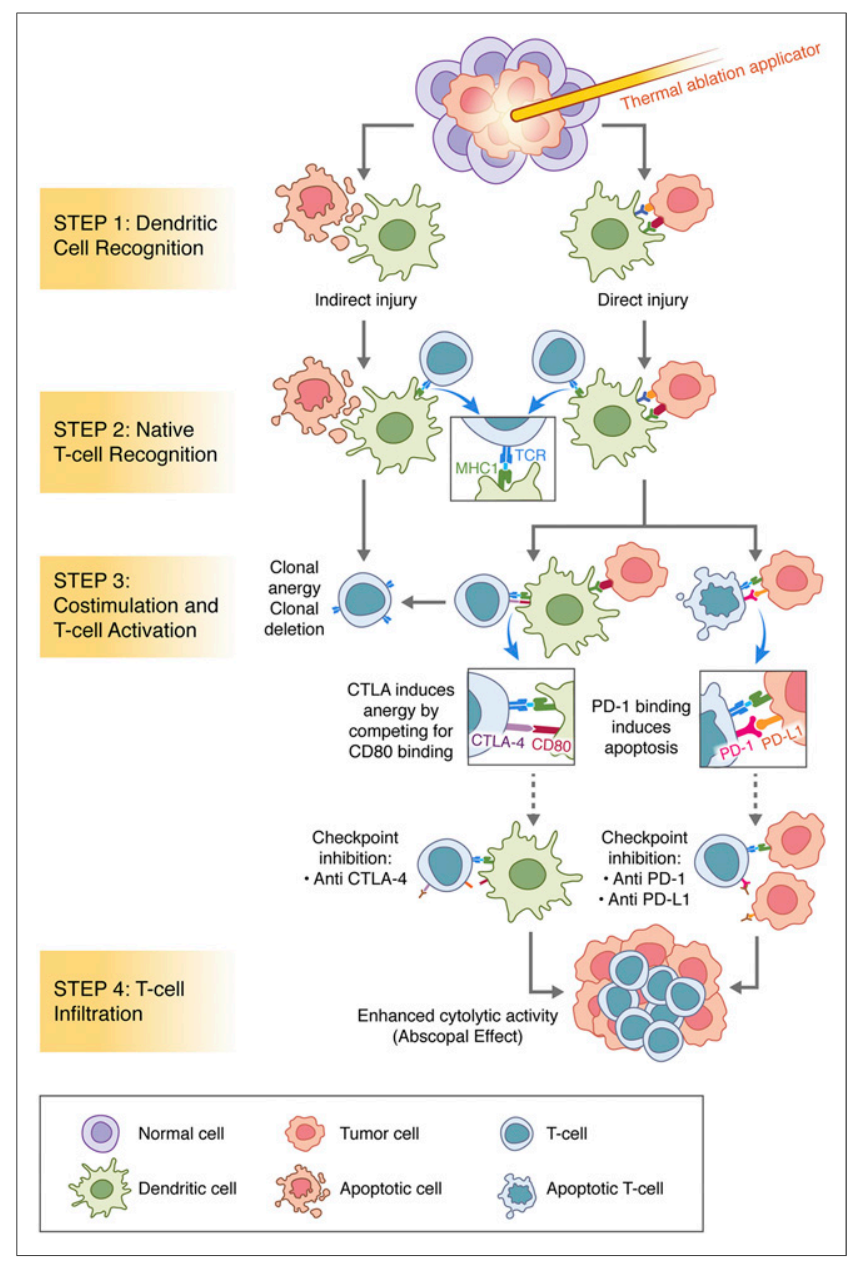

FIGURE 5. Immune pathways activated after ablation and implications for immune modulation.

remain in the preclinical stages, encouraging results have been reported in tumor models and a few clinical trials are under way $(56,57)$. Most of these trials involve the combined use of thermal ablation and immune checkpoint inhibitors such as anti-CTLA-4 (cytotoxic T-lymphocyte-associated protein 4), anti-PD-1 (programmed cell death protein 1), anti-PD-L1 (programmed deathligand 1), and anti-OX40 (or CD134 is a member of the tumor necrosis factor receptor superfamily) antibodies. A similar rationale has been advocated for combined therapy with TACE, but further evaluation is required.

\section{CONCLUSION}

The benefits associated with both percutaneous liver ablations and catheter-directed therapies are supported by numerous preclinical and clinical studies. These techniques are now widely acknowledged worldwide. However, further multiinstitutional evaluations introducing standardized protocols are needed for optimization.

\section{ACKNOWLEDGMENTS}

We thank Elena N. Petre, MD, Joseph P. Erinjeri, MD, PhD, and Govindarajan Srimathveeravalli, $\mathrm{PhD}$, for their contributions. 


\section{REFERENCES}

1. Solomon SB, Cornelis F. Interventional molecular imaging. J Nucl Med. 2016;57: 493-496.

2. Covey AM, Hussain SM. Liver-directed therapy for hepatocellular carcinoma: an overview of techniques, outcomes, and posttreatment imaging findings. AJR. 2017;209:67-76.

3. Vesselle G, Quirier-Leleu C, Velasco S, et al. Predictive factors for complete response of chemoembolization with drug-eluting beads (DEB-TACE) for hepatocellular carcinoma. Eur Radiol. 2016;26:1640-1648.

4. Kim BK, Kim SU, Park JY, et al. Applicability of BCLC stage for prognostic stratification in comparison with other staging systems: single centre experience from long-term clinical outcomes of 1717 treatment-naive patients with hepatocellular carcinoma. Liver Int. 2012;32:1120-1127.

5. Brace CL. Radiofrequency and microwave ablation of the liver, lung, kidney, and bone: what are the differences? Curr Probl Diagn Radiol. 2009;38:135143.

6. Meloni MF, Chiang J, Laeseke PF, et al. Microwave ablation in primary and secondary liver tumours: technical and clinical approaches. Int J Hyperthermia. 2017;33:15-24.

7. Vroomen LGPH, Petre EN, Cornelis FH, Solomon SB, Srimathveeravalli G. Irreversible electroporation and thermal ablation of tumors in the liver, lung, kidney and bone: what are the differences? Diagn Interv Imaging. 2017;98: 609-617.

8. Petre EN, Sofocleous C. Thermal ablation in the management of colorectal cancer patients with oligometastatic liver disease. Visc Med. 2017;33:62-68.

9. Wright AS, Sampson LA, Warner TF, Mahvi DM, Lee FT. Radiofrequency versus microwave ablation in a hepatic porcine model. Radiology. 2005;236:132-139.

10. Cornelis FH, Durack JC, Kimm SY, et al. A comparative study of ablation boundary sharpness after percutaneous radiofrequency, cryo-, microwave, and irreversible electroporation ablation in normal swine liver and kidneys. Cardiovasc Intervent Radiol. 2017;40:1600-1608.

11. Yu J, Liang P, Yu X, Liu F, Chen L, Wang Y. A comparison of microwave ablation and bipolar radiofrequency ablation both with an internally cooled probe: results in ex vivo and in vivo porcine livers. Eur J Radiol. 2011;79:124130.

12. Shady W, Petre EN, Gonen M, et al. Percutaneous radiofrequency ablation of colorectal cancer liver metastases: factors affecting outcomes-a 10-year experience at a single center. Radiology. 2016;278:601-611.

13. Higgins MCSS, Soulen MC. Combining locoregional therapies in the treatment of hepatocellular carcinoma. Semin Intervent Radiol. 2013;30:74-81.

14. Ziemlewicz TJ, Hinshaw JL, Lubner MG, et al. Percutaneous microwave ablation of hepatocellular carcinoma with a gas-cooled system: initial clinical results with 107 tumors. J Vasc Interv Radiol. 2015;26:62-68.

15. Li Z, Zhang K, Lin S-M, et al. Radiofrequency ablation combined with percutaneous ethanol injection for hepatocellular carcinoma: a systematic review and meta-analysis. Int J Hyperthermia. October 5, 2016 [Epub ahead of print].

16. Chen M-S, Li J-Q, Zheng Y, et al. A prospective randomized trial comparing percutaneous local ablative therapy and partial hepatectomy for small hepatocellular carcinoma. Ann Surg. 2006;243:321-328.

17. Chen X, Chen Y, Li Q, Ma D, Shen B, Peng C. Radiofrequency ablation versus surgical resection for intrahepatic hepatocellular carcinoma recurrence: a metaanalysis. J Surg Res. 2015;195:166-174.

18. Huang J, Yan L, Cheng Z, et al. A randomized trial comparing radiofrequency ablation and surgical resection for HCC conforming to the Milan criteria. Ann Surg. 2010;252:903-912.

19. Potretzke TA, Ziemlewicz TJ, Hinshaw JL, et al. Microwave versus radiofrequency ablation treatment for hepatocellular carcinoma: a comparison of efficacy at a single center. $J$ Vasc Interv Radiol. 2016;27:631-638.

20. Xu Y, Shen Q, Liu P, et al. Microwave ablation for the treatment of hepatocellular carcinoma that met up-to-seven criteria: feasibility, local efficacy and longterm outcomes. Eur Radiol. 2017;27:3877-3887.

21. Sotirchos VS, Petrovic LM, Gönen M, et al. Colorectal cancer liver metastases: biopsy of the ablation zone and margins can be used to predict oncologic outcome. Radiology. 2016;280:949-959.

22. Ryan MJ, Willatt J, Majdalany BS, et al. Ablation techniques for primary and metastatic liver tumors. World J Hepatol. 2016;8:191-199.

23. Chinnaratha MA, Chuang MA, Fraser RJL, Woodman RJ, Wigg AJ. Percutaneous thermal ablation for primary hepatocellular carcinoma: a systematic review and meta-analysis. J Gastroenterol Hepatol. 2016;31:294-301.

24. Livraghi T, Meloni F, Solbiati L, Zanus G; Collaborative Italian Group using AMICA system. Complications of microwave ablation for liver tumors: results of a multicenter study. Cardiovasc Intervent Radiol. 2012;35:868-874.
25. Meloni MF, Chiang J, Laeseke PF, et al. Microwave ablation in primary and secondary liver tumours: technical and clinical approaches. Int J Hyperthermia. 2017;33:15-24.

26. Idée J-M, Guiu B. Use of lipiodol as a drug-delivery system for transcatheter arterial chemoembolization of hepatocellular carcinoma: a review. Crit Rev Oncol Hematol. 2013;88:530-549.

27. Gbolahan OB, Schacht MA, Beckley EW, LaRoche TP, O’Neil BH, Pyko M. Locoregional and systemic therapy for hepatocellular carcinoma. J Gastrointest Oncol. 2017;8:215-228.

28. Llovet JM, Bruix J. Systematic review of randomized trials for unresectable hepatocellular carcinoma: chemoembolization improves survival. Hepatology. 2003;37: $429-442$.

29. Lencioni R, Crocetti L. Local-regional treatment of hepatocellular carcinoma. Radiology. 2012;262:43-58.

30. Brown KT, Do RK, Gonen M, et al. Randomized trial of hepatic artery embolization for hepatocellular carcinoma using doxorubicin-eluting microspheres compared with embolization with microspheres alone. J Clin Oncol. 2016;34:2046-2053.

31. Song JE, Kim DY. Conventional vs drug-eluting beads transarterial chemoembolization for hepatocellular carcinoma. World J Hepatol. 2017;9:808-814.

32. Pesapane F, Nezami N, Patella F, Geschwind JF. New concepts in embolotherapy of HCC. Med Oncol. 2017;34:58

33. Maluccio MA, Covey AM, Ben Porat L, et al. Transcatheter arterial embolization with only particles for the treatment of unresectable hepatocellular carcinoma. J Vasc Interv Radiol. 2008;19:862-869.

34. Llovet JM, Real MI, Montaña X, et al. Arterial embolisation or chemoembolisation versus symptomatic treatment in patients with unresectable hepatocellular carcinoma: a randomised controlled trial. Lancet. 2002;359:17341739.

35. Lammer J, Malagari K, Vogl T, et al. Prospective randomized study of doxorubicin-eluting-bead embolization in the treatment of hepatocellular carcinoma: results of the PRECISION V study. Cardiovasc Intervent Radiol. 2010;33: 41-52.

36. Aliberti C, Carandina R, Sarti D, et al. Chemoembolization with drug-eluting microspheres loaded with doxorubicin for the treatment of cholangiocarcinoma. Anticancer Res. 2017;37:1859-1863.

37. Hyder O, Marsh JW, Salem R, et al. Intra-arterial therapy for advanced intrahepatic cholangiocarcinoma: a multi-institutional analysis. Ann Surg Oncol. 2013;20: 3779-3786.

38. Ierardi AM, Angileri SA, Patella F, et al. The role of interventional radiology in the treatment of intrahepatic cholangiocarcinoma. Med Oncol. 2017;34:11.

39. Maher B, Ryan E, Little M, Boardman P, Stedman B. The management of colorectal liver metastases. Clin Radiol. 2017;72:617-625.

40. Vogl TJ, Zangos S, Eichler K, Yakoub D, Nabil M. Colorectal liver metastases: regional chemotherapy via transarterial chemoembolization (TACE) and hepatic chemoperfusion: an update. Eur Radiol. 2007;17:1025-1034.

41. Lencioni R, Aliberti C, de Baere T, et al. Transarterial treatment of colorectal cancer liver metastases with irinotecan-loaded drug-eluting beads: technical recommendations. J Vasc Interv Radiol. 2014;25:365-369.

42. Fiorentini G, Aliberti C, Tilli M, et al. Intra-arterial infusion of irinotecan-loaded drug-eluting beads (DEBIRI) versus intravenous therapy (FOLFIRI) for hepatic metastases from colorectal cancer: final results of a phase III study. Anticancer Res. 2012;32:1387-1395.

43. Pelage J-P, Fohlen A, Mitry E, Lagrange C, Beauchet A, Rougier P. Chemoembolization of neuroendocrine liver metastases using streptozocin and tris-acryl microspheres: Embozar (EMBOsphere + ZAnosaR) study. Cardiovasc Intervent Radiol. 2017;40:394-400.

44. de Baere T, Deschamps F, Teriitheau C, et al. Transarterial chemoembolization of liver metastases from well differentiated gastroenteropancreatic endocrine tumors with doxorubicin-eluting beads: preliminary results. $J$ Vasc Interv Radiol. 2008;19:855-861.

45. Marrache F, Vullierme MP, Roy C, et al. Arterial phase enhancement and body mass index are predictors of response to chemoembolisation for liver metastases of endocrine tumours. Br J Cancer. 2007;96:49-55.

46. Wang M, Zhang J, Ji S, et al. Transarterial chemoembolisation for breast cancer with liver metastasis: a systematic review. Breast. 2017;36:25-30.

47. Vogl TJ, Mack MG, Balzer JO, et al. Liver metastases: neoadjuvant downsizing with transarterial chemoembolization before laser-induced thermotherapy. Radiology. 2003;229:457-464.

48. Liapi E, Geschwind JF. Transcatheter and ablative therapeutic approaches for solid malignancies. J Clin Oncol. 2007;25:978-986.

49. Blackburn H, West S. Management of postembolization syndrome following hepatic transarterial chemoembolization for primary or metastatic liver cancer. Cancer Nurs. 2016;39:E1-E18. 
50. Schell SR, Wessels FJ, Abouhamze A, Moldawer LL, Copeland EM. Pro- and antiinflammatory cytokine production after radiofrequency ablation of unresectable hepatic tumors. J Am Coll Surg. 2002;195:774-781.

51. Yang H, Seon J, Sung PS, et al. Dexamethasone prophylaxis to alleviate postembolization syndrome after transarterial chemoembolization for hepatocellular carcinoma: a randomized, double-blinded, placebo-controlled study. J Vasc Interv Radiol. 2017;28:1503-1511.

52. Peng Z-W, Zhang Y-J, Liang H-H, Lin X-J, Guo R-P, Chen M-S. Recurrent hepatocellular carcinoma treated with sequential transcatheter arterial chemoembolization and RF ablation versus RF ablation alone: a prospective randomized trial. Radiology. 2012;262:689-700.

53. Peng Z-W, Zhang Y-J, Chen M-S, et al. Radiofrequency ablation with or without transcatheter arterial chemoembolization in the treatment of hepatocellular carcinoma: a prospective randomized trial. J Clin Oncol. 2013;31:426-432.

54. Rossmann C, McCrackin MA, Armeson KE, Haemmerich D. Temperature sensitive liposomes combined with thermal ablation: effects of duration and timing of heating in mathematical models and in vivo. PLoS One. 2017;12:e0179131.

55. Tak WY, Lin S-M, Wang Y, et al. Phase III HEAT study adding lyso-thermosensitive liposomal doxorubicin to radiofrequency ablation in patients with unresectable hepatocellular carcinoma lesions. Clin Cancer Res. 2018;24:73-83.
56. Slovak R, Ludwig JM, Gettinger SN, Herbst RS, Kim HS. Immuno-thermal ablations: boosting the anticancer immune response. J Immunother Cancer. 2017; 5:78.

57. Takaki H, Cornelis F, Kako Y, Kobayashi K, Kamikonya N, Yamakado K. Thermal ablation and immunomodulation: from preclinical experiments to clinical trials. Diagn Interv Imaging. 2017;98:651-659.

58. Velez E, Goldberg SN, Kumar G, et al. Hepatic thermal ablation: effect of device and heating parameters on local tissue reactions and distant tumor growth. $R a$ diology. 2016;281:782-792.

59. Waitz R, Solomon SB, Petre EN, et al. Potent induction of tumor immunity by combining tumor cryoablation with anti-CTLA-4 therapy. Cancer Res. 2012;72: 430-439.

60. Takaki H, Imai N, Contessa TT, et al. Peripheral blood regulatory t-cell and type 1 helper t-cell population decrease after hepatic artery embolization. $J$ Vasc Interv Radiol. 2016;27:1561-1568.

61. Erinjeri JP, Thomas CT, Samoilia A, et al. Image-guided thermal ablation of tumors increases the plasma level of interleukin-6 and interleukin-10. J Vasc Interv Radiol. 2013;24:1105-1112.

62. Chu KF, Dupuy DE. Thermal ablation of tumours: biological mechanisms and advances in therapy. Nat Rev Cancer. 2014;14:199-208. 